\title{
Single-shot characterization of independent femtosecond extreme ultraviolet free electron and infrared laser pulses
}

\author{
P. Radcliffe, a) S. Düsterer, A. Azima, H. Redlin, and J. Feldhaus \\ HASYLAB, DESY, Notkestr. 85, D-22607 Hamburg, Germany \\ J. Dardis, K. Kavanagh, H. Luna, J. Pedregosa Gutierrez, P. Yeates, \\ E. T. Kennedy, and J. T. Costello \\ National Center for Plasma Science and Technology, Dublin City University, Dublin, Ireland \\ and School of Physical Sciences, Dublin City University, Dublin 9, Ireland \\ A. Delserieys and C. L. S. Lewis \\ International Research Centre for Experimental Physics, Queen's University Belfast, BT7 1NN Northern \\ Ireland, United Kingdom \\ R. Taïeb and A. Maquet \\ Laboratoire de Chimie Physique-Matière et Rayonnement, UMR 7614, Université Pierre et Marie Curie, \\ 11 Rue Pierre et Marie Curie, 75231 Paris Cedex 05, France \\ D. Cubaynes and M. Meyer \\ LIXAM/CNRS, UMR 8624 Centre Universitaire Paris-Sud, Bâtiment 350, F-91405 Orsay Cedex, France
}

(Received 18 October 2006; accepted 19 February 2007; published online 28 March 2007)

\begin{abstract}
Two-color above threshold ionization of helium and xenon has been used to analyze the synchronization between individual pulses of the femtosecond extreme ultraviolet (XUV) free electron laser in Hamburg and an independent intense $120 \mathrm{fs}$ mode-locked Ti:sapphire laser. Characteristic sidebands appear in the photoelectron spectra when the two pulses overlap spatially and temporally. The cross-correlation curve points to a $250 \mathrm{fs}$ rms jitter between the two sources at the experiment. A more precise determination of the temporal fluctuation between the XUV and infrared pulses is obtained through the analysis of the single-shot sideband intensities. (C) 2007 American Institute of Physics. [DOI: 10.1063/1.2716360]
\end{abstract}

The free electron laser in Hamburg (FLASH) facility, which became operational in August 2005, represents a key milestone on the road map of intense short wavelength radiation generation. ${ }^{1,2}$ Based on the process of self-amplified spontaneous emission (SASE), ${ }^{3}$ the free electron laser (FEL) is a powerful source of extremely bright and ultrashort coherent laser light. With extreme ultraviolet (XUV) FEL light fundamental processes such as direct one or few photon inner shell ionizations, as well as two-color pump-probe experiments involving intense laser fields of hugely different photon energies can be explored. For the latter, the optical laser can be used to state prepare species prior to ionization in the XUV field or alternatively species may be ionized while the resultant photo- or Auger electrons are strongly coupled to the intense low frequency laser field. ${ }^{4,5}$ Importantly, since both the XUV and optical photon energy may be tuned, one or both steps can be made resonant. ${ }^{6}$

The principal challenge facing all two-color investigations is the synchronization of two independent sources, where the inherent jitter is the limiting factor on the time resolution obtainable. Several schemes have recently been developed in the optical regime achieving a relative jitter of less than a femtosecond. ${ }^{7}$ On the other hand, due to the completely different nature of the light amplification process in SASE free electron lasers compared to conventional lasers, alternative methods are necessary to synchronize the electron bunches in the accelerator where the FEL pulses are produced, with a femtosecond optical laser. In this letter, we

\footnotetext{
${ }^{a)}$ Electronic mail: paul.radcliffe@desy.de
}

present measurements of the synchronization between FEL light and an optical laser on a femtosecond time scale directly at the experimental end stations.

A two-color process which is sensitive to the separation between XUV and optical laser pulses is the dressing of continuum electrons by one or more optical photons. This occurs when electrons generated by XUV radiation are ejected into an intense optical laser field. In this case, the electrons can exchange photons with the dressing field and sidebands appear in the photoelectron spectrum. ${ }^{5,8,9}$ In this work, after interaction of the XUV light with an effusive atomic gas jet, the photoelectrons are measured by a magnetic bottle timeof-flight (TOF) spectrometer with a $4 \pi$ angular acceptance. ${ }^{10}$ Hence, the intensity of the sidebands is independent of the precise shape of the photoelectron angular distribution.

The FEL was operated at $25.5 \mathrm{~nm}(48.5 \mathrm{eV})$ and $13.8 \mathrm{~nm}$ $(89.9 \mathrm{eV})$ with a repetition rate of $5 \mathrm{~Hz}$ in single bunch mode with peak energies up to $50 \mu \mathrm{J}$, and a pulse duration of 15-20 fs estimated from the spectral width. ${ }^{2}$ A Ti:sapphire laser is used to produce ultrashort optical laser pulses, synchronized to the reference frequencies $(1.3 \mathrm{GHz}$ and $108 \mathrm{MHz}$ ) from the master oscillator driving the electron accelerator. After amplification in an optical parametric amplifier, the femtosecond pulses (120 fs, $800 \mathrm{~nm}$, and $20 \mu \mathrm{J})$ are sent to the experiments through a dedicated beam line. At the experimental chamber, the infrared (IR) beam was introduced in a collinear geometry ${ }^{11}$ and about $5 \mu \mathrm{J}$ energy contained within a focal spot of $50 \pm 10 \mu \mathrm{m}$ diameter could provide an average intensity of about $I_{\mathrm{IR}}=2 \times 10^{12} \mathrm{~W} / \mathrm{cm}^{2}$. 


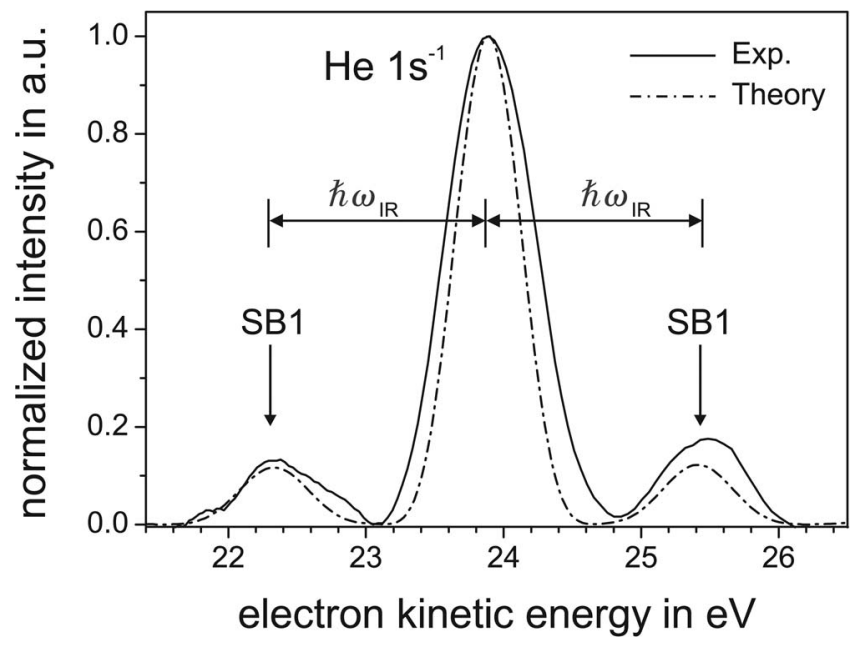

FIG. 1. Photoelectron spectrum in the region of $\mathrm{He} 1 s^{-1}$ for overlapping FEL + IR beams showing the high and low-energy sidebands (SB). (dashed line) Theoretical photoelectron spectrum of He obtained from TDSE calculations (Ref. 12) for the present characteristics of the FEL and optical laser.

The diameter of the XUV FEL beam in the interaction volume was $100 \pm 10 \mu \mathrm{m}\left(I_{\mathrm{XUV}} \approx 10^{13} \mathrm{~W} / \mathrm{cm}^{2}\right)$, hence eliminating intensity variations which could arise from spatial jitter. Spatial alignment of both lasers was achieved by monitoring, with a microscope, a phosphor screen placed at the interaction region of the TOF. The time delay between the two laser pulses was controlled by passing the IR beam through a variable delay stage. Long term drifts of up to \pm 3 ps between the two lasers were monitored by a fast streak camera with better than 1 ps (rms) resolution.

In Fig. 1, a typical single-shot two-color ionization spectrum of atomic helium recorded with the temporally and spatially overlapped FEL+IR beams is shown. The main $\mathrm{He}$ $1 s^{-1}$ photoline at $23.9 \mathrm{eV}$ results from direct photoionization by the FEL radiation at $\hbar \omega($ FEL $)=48.5 \mathrm{eV}(25.5 \mathrm{~nm})$. In addition, strong features at kinetic energies of $\pm 1.55 \mathrm{eV}$, which correspond to the emission and absorption of an additional infrared photon are observed. Previously, with $523 \mathrm{~nm}$ $(2.37 \mathrm{eV})$ picosecond laser pulses $\left(12 \mathrm{ps}, 2 \times 10^{11} \mathrm{~W} / \mathrm{cm}^{2}\right)$ a sideband magnitude of $2 \%$ of the $\mathrm{He} 1 s^{-1}$ photoline was observed. ${ }^{11}$ Using now the femtosecond laser with its higher

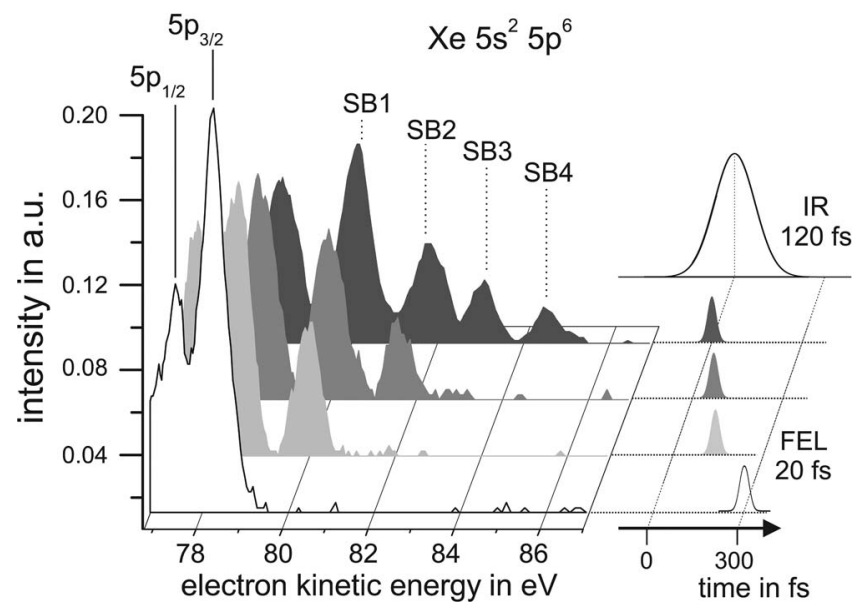

FIG. 2. Single-shot spectra in the region of the Xe $5 p^{-1}$ main line and the high-energy sidebands taken at the nominal temporal overlap $\Delta t=0$ and due to jitter also at $\Delta t \neq 0$. The spectra are normalized to total FEL + IR intensity. (right) Schematic of temporal overlap between FEL and IR pulses.

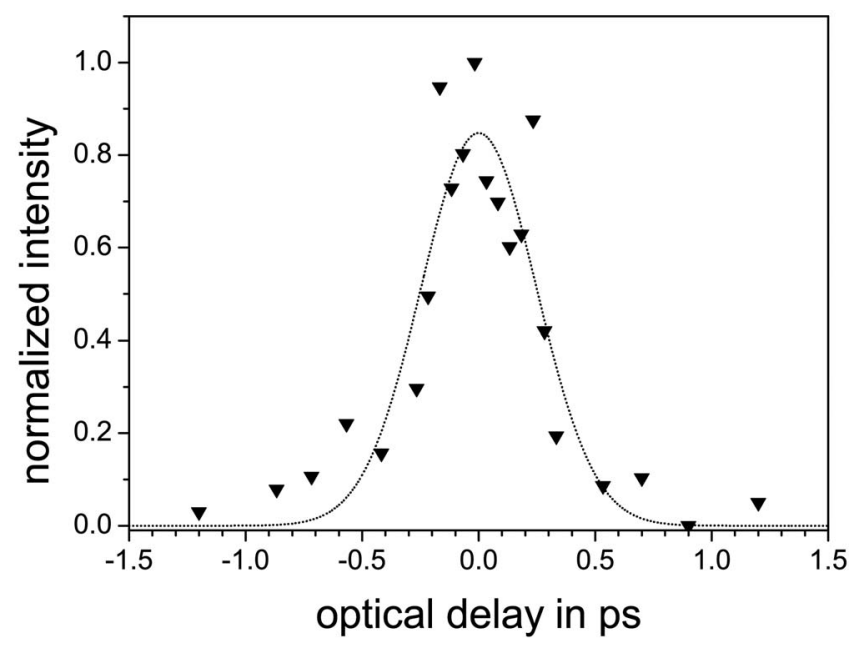

FIG. 3. Cross correlation between the pulses of the FEL (20 fs, $13.8 \mathrm{~nm}$ ) and of the IR (120 fs, $800 \mathrm{~nm}$ ) laser. Each data point represents the signal obtained when integrating over all sidebands using an average over 250 shots.

intensity, a much stronger interaction of the dressing field with the continuum electrons is induced, leading to sideband intensities of the order of $20 \%$ of the main line. Shown in Fig. 1 is the result of a theoretical analysis of the spectrum, obtained by solving the time-dependent Schrödinger equation (TDSE) for a single active electron ${ }^{12}$ using Gaussian profiles for the temporal and spatial pulse shapes of the IR and the FEL. The theoretical spectrum, taking into account the different focal spot sizes, agrees well with the measured spectrum within the limits of our experimental errors.

In Fig. 2 single-shot two-color photoionization spectra, recorded at $\hbar \omega(\mathrm{FEL})=89.9 \mathrm{eV}(13.8 \mathrm{~nm})$ with xenon, at the nominal maximum of the temporal overlap $(\Delta t=0)$ are shown. For clarity, only the main $5 p^{-1}$ spin-orbit split photolines and their high-energy sidebands are displayed. Compared to the measurement on helium (Fig. 1) the integrated sideband intensity is even more pronounced, which is mainly attributed to the fact that the sideband intensity scales like the photoelectron wave vector $k=\sqrt{2 m\left[\hbar \omega(\mathrm{FEL})-I_{p}\right]} / \hbar$, where $m$ is the electron mass and $I_{p}$ is the atomic ionization energy. The Xe spectra provide therefore a very sensitive monitor for the temporal overlap of the FEL and IR pulses. The strongest sideband intensity, i.e., observation of sidebands up to fourth order, and a remarkable bleaching of the one-color signal can only be observed for high dressing fields, i.e., for perfect overlap between both pulses. On the other hand, the absence of the sideband signal indicates a time difference larger than the width of the IR pulse. We note that the region of temporal overlap can be increased by stretching the IR pulses at the cost of sideband signal intensity. Our theoretical simulation ${ }^{13}$ for the sideband intensities show that the spectra with one or two sidebands correspond to a decrease of dressing field intensity by a factor of 3 and 12, i.e., temporal delays of 80 and $115 \mathrm{fs}$ with respect to perfect overlap at $\Delta t=0 \mathrm{fs}$. In this way, we demonstrate that it is possible to obtain via the analysis of the sideband intensity a determination of the relative temporal delay between individual independent FEL and IR pulses with a precision of better than $50 \mathrm{fs}$, however, with this analysis it is not possible to determine which of the pulses comes first. 


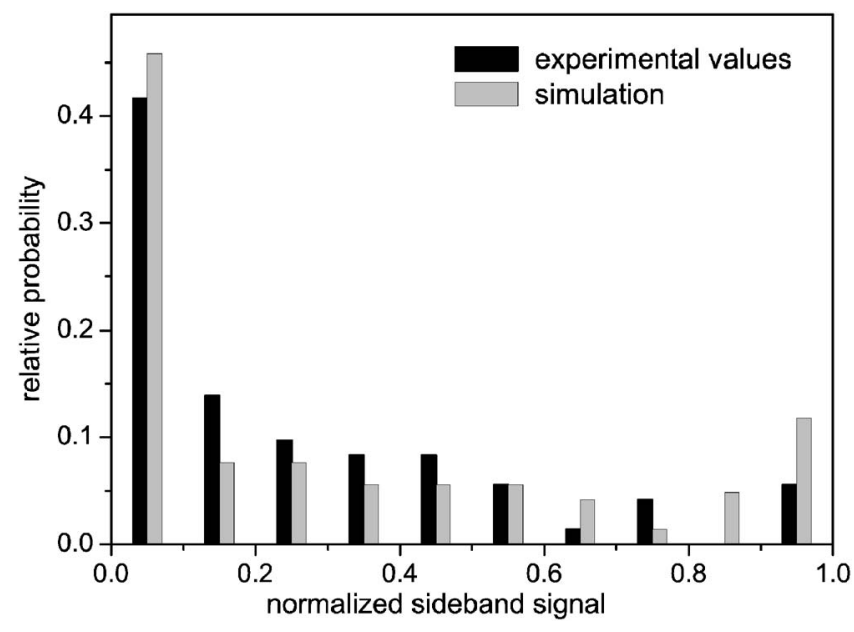

FIG. 4. Histogram of the relative probability distribution of the single-shot sideband signals. The simulation (gray) uses the experimentally determined jitter (250 fs).

The strong intensity variations in the single-shot spectra underline the importance of the temporal fluctuation between the XUV and IR pulses. Knowledge of this jitter, due to imperfect synchronization of the optical laser to the reference frequency of the accelerator and fluctuations in the arrival time of the electron bunch at the undulator, ${ }^{14}$ is a mandatory requirement for time-resolved experiments at FLASH. In order to determine its magnitude, we performed a crosscorrelation scan between the FEL and the optical laser by changing the optical delay via a translational stage over the range of \pm 1 ps while recording the sum of all sidebands at each delay over 250 FEL pulses. The sideband signal as a function of delay is shown in Fig. 3. A Gaussian profile can be fitted to the data points with a full width half maximum (FWHM) of $600 \pm 50$ fs. Given the temporal widths of the optical laser (120 fs) and of the even shorter FEL (20 fs) pulses, the dominating contribution is attributed, as expected, to the jitter of the system. Deconvolution of the crosscorrelation curve results in a value of $250 \mathrm{fs}$ rms (590 fs FWHM) for the overall jitter between the FEL and optical pulses. This value is corroborated by similar values, which have been determined in another experiment using an electro-optic sampling technique. ${ }^{14}$

The shot-to-shot fluctuation between the two pulses and its statistical behavior are obtained by comparing the individual spectra with a simulation of the sideband amplitudes, calculated in arbitrary units from the overlap $I_{\mathrm{SB}}$ $\sim \int I_{\mathrm{opt}}(t) I_{\mathrm{FEL}}\left(t-t_{j}\right) d t$. Where $I_{\mathrm{FEL}}$ and $I_{\mathrm{opt}}$ are the Gaussian intensity profiles of the FEL and the optical laser, respectively, and $t_{j}$ is the jitter simulated by a random generator (see Fig. 4). The only input parameters are the measured pulse duration of both sources and the Gaussian jitter (250 fs rms), as determined experimentally. From Fig. 4 the following can be determined: Averaging over the temporal fluctuations yields $20 \%-25 \%$ of the signal obtained compared to the optimum overlap (weighted average of the relative prob- ability, Fig. 4). Whereas recording single-shot data, which is essential in order to study intensity dependent phenomena, will yield about 5\%-10\% perfect overlap, however, approximately $40 \%-45 \%$ will have no cross correlation at all.

In conclusion, prominent two-color multiphoton photoionization signatures were used to characterize the individual FEL and optical laser pulses with respect to their spatial and temporal overlaps. Up to four sidebands have been observed for the excitation of Xe at $13.8 \mathrm{~nm}$. In average mode a time resolution of $250 \mathrm{fs}$ rms was achieved, which corresponds to the jitter of the system. Numerical simulations combined with single-shot sideband intensity data yield a precision of better than $50 \mathrm{fs}$ provided both pulses are temporally overlapped.

We are greatly indebted to the scientific and technical team at FLASH, in particular the machine operators and run coordinators, being the foundation of the successful operation and delivery of the SASE-FEL beam. Support from the EU RTD project "X-Ray FEL pump probe" (HRPI-CT-199950009) is acknowledged. Support from the SFI Frontiers (DCU researchers) and HEA North-South (DCU and QUB researchers) schemes is also acknowledged. ${ }^{1}$ J. Andruszkow et al., Phys. Rev. Lett. 85, 3825 (2000).
${ }^{2}$ V. Ayvazyan et al., Eur. Phys. J. D 37, 297 (2006).
${ }^{3}$ E. Saldin, E. Schneidmiller, and M. Yurkov, The Physics of Free Electron
Lasers (Springer, New York, 1999), Chap. 6, p. 353 .
${ }^{4}$ J. M. Schins, P. Breger, P. Agostini, R. C. Constantinescu, H. G. Muller,
G. Grillon, A. Antonetti, and A. Mysyrowicz, Phys. Rev. Lett. 73, 2180
(1994).

${ }^{5}$ T. E. Glover, R. W. Schoenlein, A. H. Chin, and C. V. Shank, Phys. Rev. Lett. 76, 2468 (1996).

${ }^{6}$ S. I. Themelis, P. Lambropoulos, and M. Meyer, J. Phys. B 37, 4281 (2004); S. I. Themelis, P. Lambropoulos, and F. J. Wuilleumier, ibid. 38, 2119 (2005).

${ }^{7}$ T. R. Schibli, J. Kim, O. Kuzucu, J. T. Gopinath, S. N. Tandon, G. S. Petrich, L. A. Kolodziejski, J. G. Fujimoto, E. P. Ippen, and F. X. Kaertner, Opt. Lett. 28, 947 (2003).

${ }^{8}$ E. S. Toma, H. G. Muller, P. M. Paul, P. Breger, P. Agostini, C. Le Blanc, G. Mullot, and G. Cheriaux, Phys. Rev. A 62, 061801(R) (2000).

${ }^{9}$ P. O'Keeffe, R. López-Martens, J. Mauritsson, A. Johansson, A. L'Huillier, V. Véniard, R. Taïeb, A. Maquet, and M. Meyer, Phys. Rev. A 69, 051401(R) (2004).

${ }^{10}$ S. Düsterer, P. Radcliffe, G. Geloni, U. Jastrow, M. Kuhlmann, E. Plönjes, K. Tiedtke, R. Treusch, J. Feldhaus, P. Nicolosi, L. Poletto, P. Yeates, H. Luna, J. T. Costello, P. Orr, D. Cubaynes, and M. Meyer, Opt. Lett. 31, 1750 (2006).

${ }^{11}$ M. Meyer, D. Cubaynes, P. O’Keeffe, H. Luna, P. Yeates, E. T. Kennedy, J. T. Costello, P. Orr, R. Taïeb, A. Maquet, S. Düsterer, P. Radcliffe, H. Redlin, A. Azima, E. Plönjes, and J. Feldhaus, Phys. Rev. A 74, 011401(R) (2006).

${ }^{12}$ O. Guyétand, M. Gisselbrecht, A. Huetz, P. Agostini, R. Taïeb, V. Véniard, A. Maquet, O. Boyko, C. Valentin, and D Douillet, Opt. Phys. 38, L357 (2005).

${ }^{13}$ The simulation was performed by solving the time-dependent Schrödinger equation for a "single active electron" xenon atom with a laser intensity of $10^{12} \mathrm{~W} \mathrm{~cm}^{-2}$

${ }^{14}$ A. Azima, S. Düsterer, H. Schlarb, J. Feldhaus, A. L. Cavalieri, D. Fritz, and K. Sengstock, EPAC Conference Proceeding 2006, Edinburgh (European Physical Society Accelerator Group, 2006) p. 71. 